\title{
Structural and functional changes in glaucoma: comparing the two-flash multifocal electroretinogram to optical coherence tomography and visual fields
}

\author{
Anna A. Ledolter • Matthias Monhart • \\ Andreas Schoetzau • Margarita G. Todorova • \\ Anja M. Palmowski-Wolfe
}

Received: 29 October 2013/Accepted: 15 January 2015/Published online: 24 January 2015

(C) Springer-Verlag Berlin Heidelberg 2015

\begin{abstract}
Purpose To correlate multifocal electroretinogram (mfERG) findings in the macular area of glaucoma patients with automated perimetry (visual fields) and with optical coherence tomography (OCT).

Methods A two-global flash mfERG (VERIS ${ }^{\mathrm{TM}}$ ) was recorded in 20 eyes with primary open-angle glaucoma. The root mean square was calculated, and three response epochs were analysed: the direct component (15-45 ms) and two induced components (IC-1 at 45-75 ms and IC-2 at 75-105 ms). The central $10^{\circ}$ of the mfERG was compared to the central $10^{\circ}$ of the OCT and of the visual field. Responses grouped in a superior and in an inferior semicircle, extending between $10^{\circ}$ and $20^{\circ}$, were also compared to the corresponding areas of the OCT and of the visual
\end{abstract}

The results of this manuscript have been presented in part at the ISCEV Symposium 2012, Valencia, Spain.

A. A. Ledolter - A. Schoetzau · M. G. Todorova

A. M. Palmowski-Wolfe

Department of Ophthalmology, University of Basel,

Basel, Switzerland

\section{A. A. Ledolter $(\square)$}

Department of Ophthalmology, Medical University of Vienna, Währinger Gürtel 18-20, 1090 Vienna, Austria e-mail: anna.ledolter@meduniwien.ac.at

M. Monhart

Carl Zeiss AG, Feldbach, Switzerland fields. In addition, the area of the papillomacular bundle was also analysed separately.

Results In glaucoma patients, mfERG responses showed a significant positive association with retinal thickness in the central $10^{\circ}$ for IC2 $(p=0.001)$ and a trend for IC1 $(p=0.066)$. A significant association was found between the central IC1 and IC2 of the mfERG and corresponding perimetric sensitivities expressed in linear units $(p<0.01)$. The OCT showed a positive association with visual field sensitivities $(p<0.05)$ in all areas examined $(p<0.05)$. Separation of the papillomacular bundle area did not improve structure-function association further.

Conclusions In our study, mfERG showed a statistically significant correlation with perimetric sensitivity measured in linear units and with structural macular changes detected with time-domain OCT.

Keywords Optical coherence tomography · Multifocal ERG · Global flash multifocal ERG · Glaucoma $\cdot$ Structure-functional relations . Visual field

\section{Introduction}

Glaucoma is a neurodegenerative disease [1], which is associated with both progressive loss of retinal function and increasing morphological changes in the retina and the optic nerve. However, these changes are not always detected concurrently [2]. Preperimetric 
glaucoma could be an example of a subgroup of glaucoma patients where identification of structural damage precedes detection of functional changes.

The macula is an area of great interest not only because of its importance in visual function but also because of its particular anatomical structure. In glaucoma, damage first occurs in the retinal ganglion cells (RGCs) and their axons. Because the macula is rich in RGCs, it seems likely that glaucomatous damage might be seen early on in the disease process in this central region [3]. While Snellen acuity seems to be an insufficient measure of central function, abnormalities of the central contrast sensitivity and defects of colour vision have been reported in patients with ocular hypertension and glaucoma [4-6].

Structural damage can be assessed with imaging devices from which the thickness of the retinal nerve fibre layer (RNFL) can be estimated. Optical coherence tomography (OCT) generates images by measuring the echo time delay of the light reflected and backscattered from ocular tissues [7]. OCT is capable of objectively quantifying the RNFL, which diminishes as RGCs are lost in glaucoma progression. Nowadays OCT is widely used in clinical practices for managing glaucoma and other retinal and optic nerve diseases. In OCTs of glaucoma patients, the optic nerve head parameters are primarily analysed. Recently, however, the role of macular OCT parameters in the detection of early glaucoma is being increasingly studied [8-12].

The loss of visual function in glaucoma is also routinely assessed with static automated perimetry (SAP). SAP provides global indices concerning the visual field and estimates of visual sensitivities at individual locations. Loss of macular function in glaucoma can also be documented with SAP. Indeed, there are some patients with early glaucoma who show initial arcuate defects within the central $10^{\circ}$ [9].

Multifocal electroretinography (mfERG) has been studied for the past decade as a possible objective diagnostic tool to detect early glaucomatous dysfunction. In experimental glaucoma, retinal ganglion cell damage induced in the primate results in a marked reduction in amplitude in the mfERG [13-17]. In humans, initial studies that describe changes in the mfERG secondary to glaucoma show only a small reduction in amplitude and an increase in latencies in primary open-angle glaucoma (POAG) patients when compared to a control group. With a mfERG using long $m$-stimulus steps of 100-ms duration, glaucoma patients differed significantly from controls, predominantly in the central $20^{\circ}$ when the latencies of the mfERG N2-on response were analysed [18]. Global flashes introduced into the stimulus sequence increased the mfERG's sensitivity to detect retinal dysfunction in glaucoma [19-22]. The mfERG response obtained with the global flash paradigm contains a direct component (DC) as a response to the focal flash of the $m$-sequence step and induced components (ICs) as a response, induced by the influence of the preceding focal flash on the response to the following global flashes within the $m$-sequence step. The induced components are thought to be generated by inner retinal components and have been reported to be affected in glaucoma in humans and animals [20, 23]. In our previous study, two-global flash mfERG responses in glaucoma patients differed significantly from controls in the central $15^{\circ}$ diameter [24]. Here, the DC differed least and the IC1 most when POAG was compared to control, where the most sensitive differential measure was IC1 of the central $10^{\circ}$.

Comparing measures of retinal structure and function in glaucoma is important not only for understanding the nature and stage of the disease, but also for recognizing deterioration of the damage or to determine the effectiveness of treatment in an individual patient.

Recently, many studies have been published concerning the association between structural and functional measures [10, 25-30]. Most of these compare the morphological changes seen on OCT to subjective functional assessments in SAP.

A few papers also address objective functional assessments such as the mfERG in comparison with the OCT. In neuroophthalmological patients, there are examples where both OCT and mfERG correlate, but also to a rather high percentage where the OCT but not the mfERG is abnormal and vice versa: for instance, out of 198 eyes, with a pathologic visual field, $26 \%$ had a retinal problem based on mfERG and/or frequencydomain OCT (fd-OCT). About $38.5 \%$ of these eyes had an abnormal mfERG but a normal fd-OCT, while $13.5 \%$ had a normal mfERG with an abnormal fd-OCT [31]. Thus, to date it is not clear how structural measures relate to functional measures and which appears most sensitive to detect early dysfunction. At the same time, the preferred method to detect progression of disease and to use for follow-up is unclear.

Thus, structure-function relationships in POAG are still a subject of continuing research [32]. The 
questions that need to be answered are how structural measures relate to functional measures and which test appears most sensitive to detect early dysfunction, especially when considering the variability that accompanies commonly used functional and structural tests.

In the present study, we relate two-global flash mfERG findings in glaucoma patients to SAP and to structural measurements made using a time-domain OCT, analysing the macula area.

\section{Patients and methods}

The study protocol was approved by the Ethics Committee of the University of Basel. Informed consent was obtained from all participants before the commencement of the examination. Procedures adhered to the tenets of the Declaration of Helsinki.

Twenty eyes from 19 POAG patients [nine eyes with normal-tension glaucoma (NTG), seven-hightension glaucoma (HTG) and four-preperimetric glaucoma (PPG)] were examined. A two-global flash mfERG, a time-domain OCT and SAP were obtained in every study eye.

Inclusion criteria: a visual acuity of 0.8 or better and a refractive error less than \pm 6 dioptres. All patients included in this study had glaucomatous optic neuropathy based on a clinical fundus examination and on the OCT, i.e. a localized thinning of the peripapillary nerve fibre layer. Patients with normal- and hightension glaucoma had an abnormal visual field (VF) on SAP. The RNFL was evaluated on OCT at a distance of 3.4-mm-diameter circle centred on the optic nerve head. This circle was divided into 12 clock-hour RNFL sectors: patients were included if one sector was $<1 \%$ (marked red) or two were $<5 \%$ (yellow) of normal [33].

Exclusion criteria: history of surgical treatment (including cataract surgery) on the examined eye, clinical signs of macular pathology, systemic diseases (such as diabetes mellitus or congenital disorders), history of antidepressants, alcohol or drugs.

\section{Multifocal electroretinogram (mfERG)}

The mfERG testing was performed using VERIS ${ }^{\mathrm{TM}}$ 6.0. (Electro-Diagnostic Imaging, San Mateo, CA, USA).
Pupils were maximally dilated with tropicamide $0.5 \%$ and phenylephrine $1 \%$ (Hospital Pharmacy, Basel, Switzerland). Responses were recorded monocularly with a standard Burian-Allen bipolar electrode (Hansen Ophthalmic Development Laboratories, Iowa City, IA, USA). The cornea was anaesthetized with tetracaine $1 \%$ (Novartis Pharma, Basel, Switzerland). Before setting the contact lens electrode into the eye, Methocel $2 \%$ (OmniVision, Neuhausen, Switzerland) was applied to the inner surface of the lens. During the test, the subject's eye was wetted with Celluvisc $1 \%$ (Allergan, Irvine, CA, USA) drops. The subjects viewed the stimulus through the VERIS refractor/camera FMS III, which was placed directly in front of the examined eye. Subjects refracted themselves to the stimulus. The visual stimulus consisted of 103 hexagons (stretch factor 10.46) covering the central $50^{\circ}$ diameter of the retina. Each hexagon flickered between black and white according to a binary $m$-sequence of $213-1$. The flash intensity was $100 \mathrm{~cd} / \mathrm{m}^{2}$, and the intensity of dark frames was $<1 \mathrm{~cd} / \mathrm{m}^{2}$. Each $m$-sequence was followed by two-global flashes with an intensity of $200 \mathrm{~cd} / \mathrm{m}^{2}$ at an interval of $26.6 \mathrm{~ms}$. Sixteen segments of $43-\mathrm{s}$ duration (11 $\mathrm{min} 28 \mathrm{~s}$ total duration) were recorded per eye. Segments of bad quality were discarded and re-recorded. Three response epochs were analysed: the direct component (DC) (15-45 ms) and two induced components [first (IC-1) at 45-75 ms and second (IC2) at 75-105 ms]. For each, DC, IC1 and IC2, focal responses were calculated as RMS with VERIS ${ }^{\mathrm{TM}}$ Science 6.2.2 d2. Average RMS was calculated for each area examined [Figs. 1, 2 (mfERG)].

\section{Optical coherent tomography (OCT)}

Macular thickness measurements were obtained with the time-domain Stratus OCT-3 (Carl Zeiss Meditec, Dublin, CA, USA). Macular measurements consisted of a series of six equally spaced line scans through a common central axis. The diameter of the aiming circle (and thus line length) is fixed at $6 \mathrm{~mm}$. Three concentric circles divide the macular thickness map into three zones: fovea, inner macula and outer macula. Inner and outer zones were divided into four segments each by two diagonal lines, thus resulting in nine areas available for analysis. These were calculated automatically by the Stratus OCT software (Carl Zeiss, Software 5.0.1) [34]. Average total macular 
mfERG



OCT

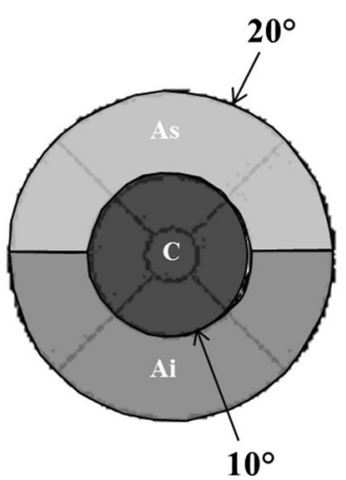

SAP



$10^{\circ}$
Fig. 1 An approximation of an overlay of the 103 focal mfERG stimulus areas (retinal view) and the group averages formed to analyse the mfERG (retinal view), the OCT (retinal view) and the visual fields (SAP, field view). The retinal nerve fibre layers leading to the optic disc are depicted in the background of

mfERG focal stimulus (modified from http://cuth.cataegu.ac.kr/ $\sim$ jwkim/glaucoma/doctor/relationship.htm). $C$ central $10^{\circ}$ from central fixation, $A s$ superior macular area, $A i$ inferior macular area

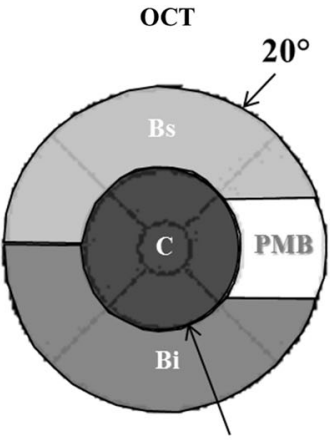

$10^{\circ}$

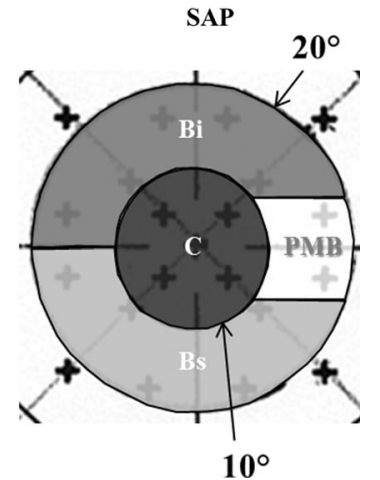

Fig. 2 Group averaging with separate analysis of the papillomacular bundle area. $C$ central $10^{\circ}$ from central fixation, $B s$ superior macular area, $B i$ inferior macular area, $P M B$ papillomacular bundle

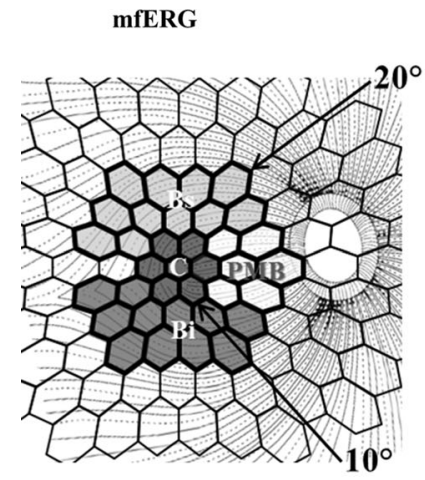

and temporal outer segments, considering that $A i$ includes one-half of the nasal outer segment, whole inferior outer segment and one-half of the temporal outer segment (Fig. 1).

B: A separate analysis included the PMB separately as shown in Fig. 2. To calculate the average retinal thickness of the superior and inferior semicircle, each outer segment was divided into six equal parts. Thus, $B s$ included six parts of the superior outer segment, three parts of the temporal outer segment and one part of the nasal outer segment. Correspondingly, $B i$ : six parts of the inferior outer segment, three parts of the temporal outer segment and one part of the nasal segment. The weighted retinal thickness averages for $B s$ and $B i$ were calculated. Average retinal thickness of the PMB was approximately equal to the average retinal thickness of the nasal outer segment. 
Visual field test (SAP)

Visual field tests were performed with the Octopus 101 G2 program (Haag-Streit AG, Köniz, Switzerland). This program has 59 test locations in the central $30^{\circ}$ with a higher density of test locations towards the macula [35]. We applied a full threshold strategy with target size III. The stimulus duration was $0.1 \mathrm{~s}$ (Octopus Standard). Every stimulus intensity was calculated in $\mathrm{dB}$ and also converted individually into linear units. For each area, the test locations were averaged in the linear space to calculate sensitivities [Figs. 1, 2 (SAP)].

\section{Comparison of the techniques}

In earlier studies [18, 22], we observed that the mfERG in glaucoma differed significantly from normal in responses from the central retina but not in those from more peripheral retina. Thus, we concentrated our analysis on the central $20^{\circ}$ of the macula, which is also still covered by the time-domain OCT. Response averages were formed, taking into consideration the anatomical and physiological structure of the nerve fibre layer: for the area of the central $10^{\circ}$ diameter and for two surrounding hemispheres extending between $10^{\circ}$ and $20^{\circ}$ inferior and superior to the centre (Fig. 1). Because of the structural specificity, we also analysed the structural-functional relations in the papilomacular bundle (PMB) area between all three methods (Fig. 2). The refractive error of the eye has been corrected in all of three methods we used: spherical equivalent for OCT and mfERG, and full proper correction to the distance on SAP.

\section{Statistical analysis}

A linear-mixed effects model was performed for each pair of tested methods, where one parameter of the tested pair is a dependent variable. Age, gender, location average, epoch of the mfERG, macular thickness, VF parameter and study groups were treated as covariates. Potential interactions between study groups and the covariates were also included in the regression model (ANOVA interactions). Subject was treated as a random factor. Results are presented as regression coefficients with corresponding $95 \%$ confidence intervals and $p$ values. For metric variables, coefficients represent the increase in the dependent variable, increasing the predictor by one unit (Table 1). For instance, increase in the mfERG response is associated with an increase in macular thickness of $1 \mu \mathrm{m}$. A $p$ value $<0.05$ was considered significant. A $p$ value $<0.1$ was treated as a trend. In order to adjust $p$ values for multiple comparisons, false discovery rate (FDR) adjustment was done.

\section{Results}

We examined a total of 20 eyes of 19 patients with POAG (nine eyes with NTG, seven-HTG and four$\mathrm{PPG}$ ). The mean $\pm \mathrm{SD}$ age was $64.8 \pm 6.7$, ranging from 53 to 81 . Demographic data are presented in Table 2. The range of refractive error of study eyes was $0-4.75$ dioptres. The refractive error of the study eye of each subject was corrected for during SAP, OCT and mfERG testing.

\section{mfERG versus OCT}

The relationship between mfERG RMS responses and macula thickness measured with OCT depended on the macular location [ANOVA interaction: $p=0.001$ (Table 3)]. Their correlation was significant in the central $10^{\circ}[p=0.001$ (FDR 0.003) $]$ and in the PMB area $[p=0.026$ (FDR 0.052)], but not in the other macular response averages analysed $[p>0.05$ (FDR $>0.063)$.

In the central $10^{\circ}, \mathrm{IC} 2$ of the mfERG had a significant positive association with the OCT $[p=0.001$ (FDR 0.003)] (Table 4 for details). A trend in the association of OCT and mfERG was observed in IC1 $[p=0.07$ (FDR 0.084)]. That is, mfERG responses were reduced when retinal thickness decreased.

\section{OCT versus VF}

The OCT showed decreasing retinal thickness with decreasing mean sensitivity for all clusters analysed $[p<0.001$ (FDR $<0.003)]$. Again, there was a strong dependency on the location [ANOVA interaction: $p<0.05$ (Table 3)].

\section{mfERG versus VF}

The ANOVA did not show a clear significance between the macular locations for the relationship 
Table 1 Indication of variables for statistical analysis

\begin{tabular}{lll}
\hline Variable & Dependent & Independent (predictor) \\
\hline Parameter of tested methods & mfERG response & Macular thickness \\
& VF sensitivity (linear) & Macular thickness \\
& mfERG response & VF sensitivity (linear) \\
\hline
\end{tabular}

Table 2 Demographic data for POAG patients

\begin{tabular}{lllllll}
\hline Demographic data & Total $(n=20)$ & \multicolumn{2}{l}{ Type of glaucoma } & & VF defect within the central 20 \\
\hline Gender (male/female) & $16 / 3$ & $6 / 2$ & $6 / 1$ & $4 / 0$ & $5 / 0$ & $12 / 3$ \\
Age (years) & $64.8 \pm 6.7$ & $63.4 \pm 6.3$ & $67.3 \pm 8.4$ & $63.5 \pm 4.2$ & $68.0 \pm 8.5$ & $63.7 \pm 6.0$ \\
Corrected visual acuity & $0.9 \pm 0.1$ & $0.96 \pm 0.7$ & $0.89 \pm 1.2$ & $0.95 \pm 0.1$ & $0.96 \pm 0.1$ & $0.92 \pm 0.1$ \\
Intraocular pressure (mmHg) & $12.1 \pm 2.3$ & $12.0 \pm 1.8$ & $11.7 \pm 3.1$ & $13.0 \pm 1.8$ & $12.0 \pm 2.0$ & $12.1 \pm 2.4$ \\
Cup/disc ratio & $0.8 \pm 0.1$ & $0.8 \pm 0.2$ & $0.7 \pm 0.1$ & $0.8 \pm 0.1$ & $0.8 \pm 0.1$ & $0.8 \pm 0.1$ \\
Octopus MD (dB) & $4.4 \pm 4.3$ & $4.9 \pm 4.2$ & $5.8 \pm 4.7$ & $0.7 \pm 0.3^{*}$ & $1.9 \pm 1.8^{* *}$ & $5.2 \pm 4.6$ \\
\hline
\end{tabular}

Values are mean $\pm \mathrm{SD}$

$n$ eyes examined, $V F$ visual field

$* p<0.05$ - significantly differed from NTG and HTG patients

$* * p<0.05$ - significantly differed from group with VF defect

Table 3 ANOVA interaction for the relation between the OCT, mfERG and SAP depends on the macular locations analysed

\begin{tabular}{lllll}
\hline Test methods & \multicolumn{2}{l}{ Macular thickness (OCT) } & & \multicolumn{2}{l}{ Visual field sensitivity } \\
\cline { 2 - 3 } & $F$ value & & & $F$ value \\
\hline Multifocal ERG & 4.19 & $<0.001$ & 1.42 & 0.216 \\
Macular thickness (OCT) & $\mathrm{n} / \mathrm{a}$ & $\mathrm{n} / \mathrm{a}$ & 2.35 & 0.041 \\
\hline
\end{tabular}

between mfERG RMS responses and VF sensitivity [ANOVA interaction: $p=0.2$ (Table 3)]. However, when all the POAG patients were analysed together, VF sensitivity had a significant negative association with mfERG parameters in the central $10^{\circ}$ for IC 1 and IC2 $[p=0.007$ (FDR 0.017) and $p<0.001$ (FDR 0.003 ), respectively] (Table 4), but no significance for DC $(p=0.74)$. We did not find any significance in the other macular response averages analysed $[p>0.05$ $($ FDR $>0.063)]$.

Structure-function relations and the severity of glaucoma

In order to better assess structure-function relationship in glaucoma and to see whether the structurefunction relations are influenced by the severity of glaucoma, we subdivided the patients' eyes into two groups according to their functional ability, tested with the current "gold-standard" SAP in the analysed area.

Seventy-five percentage ( 15 eyes) of our glaucoma patients showed an abnormal VF within the central $20^{\circ}$. For this subgroup, the mean $\pm \mathrm{SD}$ MD was $5.2 \pm 4.6 \mathrm{~dB}$. This differed significantly from the mean MD of the $25 \%$ (5 eyes) of POAG patients, who had a normal VF in the central $20^{\circ}(p<0.05)$. Four of these had a preperimetric glaucoma. The mean $\pm \mathrm{SD}$ $\mathrm{MD}$ in this group was $1.9 \pm 1.8 \mathrm{~dB}$ (Table 2).

In the group with a VF defect within the central $20^{\circ}$, central macular sensitivity in the linear range was associated significantly with IC1 and IC2 of the mfERG [ $p=0.006$ (FDR 0.016) and $p<0.001$ (FDR 0.003), respectively]. In this group, the OCT showed positive slopes with all macular cluster's sensitivities $[p<0.001$ 
Table 4 Relations between the methods analysed for central $10^{\circ}$ location

\begin{tabular}{|c|c|c|c|c|}
\hline \multirow[t]{2}{*}{ Test methods } & \multicolumn{2}{|c|}{ Macular thickness (OCT) } & \multicolumn{2}{|c|}{ Visual field sensitivity } \\
\hline & $\begin{array}{l}\text { Regression } \\
\text { coefficient }\end{array}$ & $p$ value & $\begin{array}{l}\text { Regression } \\
\text { coefficient }\end{array}$ & $p$ value \\
\hline Multifocal ERG & 0.67 & $0.001 *$ & -0.0014 & $<0.01 *$ \\
\hline \multicolumn{5}{|l|}{ Epoch of mfERG } \\
\hline $\mathrm{DC}$ & 0.03 & 0.1 & $<0.01$ & 0.74 \\
\hline IC1 & 0.04 & 0.07 & -0.002 & $<0.01^{*}$ \\
\hline IC2 & 0.08 & $0.001 *$ & -0.002 & $<0.001 *$ \\
\hline Macular thickness (OCT) & $\mathrm{n} / \mathrm{a}$ & $\mathrm{n} / \mathrm{a}$ & 25.7 & $<0.001 *$ \\
\hline
\end{tabular}

Regression coefficient represents the increase in the dependent variable, increasing the predictor by one unit. (Example for pair mfERG and macular thickness: increase in the mfERG response is associated with an increase in macular thickness of $1 \mu \mathrm{m}$ )

* Significance after false discovery rate (FDR) adjustment

(FDR 0.003)]. Also, the association between OCT and mfERG responses was significant in the central $10^{\circ}$ $[p<0.001$ (FDR 0.003)], as well as in surrounding locations $[p<0.01$ (FDR 0.022)] when all mfERG epochs were pooled together. If each mfERG epoch was analysed separately, the association between macular thickness and mfERG was observed in all locations for IC1 $[p<0.05$ (FDR $<0.057)]$ and IC2 $[p<0.01$ (FDR $<0.022)]$, for DC only in central $10^{\circ}[p=0.03$ (FDR 0.055); Fig. 3].

In 5 patients with a normal central VF, we did not observe any significant association between OCT and VF $[p>0.4$ (FDR 0.4)]. The association between OCT and mfERG responses (all epochs pooled together) was not significant in the central $10^{\circ}$ $[p=0.35$ (FDR 0.37)], but was significant in the surrounding locations $[p<0.01 \quad$ (FDR $<0.02)]$. When each mfERG epoch was analysed separately, a significant association between macular thickness and mfERG was observed in the surrounding locations for IC1 $[p<0.05(\mathrm{FDR}<0.055)]$, but not for IC2 $[p>0.05(\mathrm{FDR}>0.063)]$ and DC $[p>0.05$ (FDR $0.063)]$. In the central $10^{\circ}$, we did not find any significant association with any of the three epochs [ $p>0.2$ (FDR 0.22); (Fig. 3)].

Thus, the relation between mfERG response and macular thickness depends not only on the location within the macula, but also on the severity of disease. Induced components of the mfERG, which are supposed to mainly consist of inner retinal contributions (IC1 and IC2) [23], have a stronger association in more advanced glaucoma. In early glaucoma that is in the group without central field defects, IC1 of mfERG showed more association with macular thickness, than DC and IC2.

Papillomacular bundle

Separation of the PMB area did not improve structurefunction association further. In the PMB area itself, a significant association was seen between macular thickness and VF parameters $[p<0.001$ (FDR $<0.003)]$ in the group of advanced glaucoma, and between macular thickness and mfERG response in both subgroups $[p<0.01$ (FDR $<0.02)$, with a stronger association in the subgroup with a central VF defect. There was no significant association between mfERG responses and VF parameters $[p>0.1($ FDR $>0.11)]$.

\section{Discussion}

OCT offers a fast and reproducible method to assess retinal morphology. The question has arisen whether the OCT can replace functional tests, which take longer to obtain, such as the mfERG [36]. In the present study, we compare a patient's two-global flash mfERG, time-domain OCT macular thickness measurements and VF in a group of POAG patients.

Recent studies in glaucoma have shown that morphologic changes are also found in the macular area and that a macular OCT may be even more sensitive in assessing progressive glaucomatous retinal damage than measurements of the peripapillary nerve fibre layer [37]. 


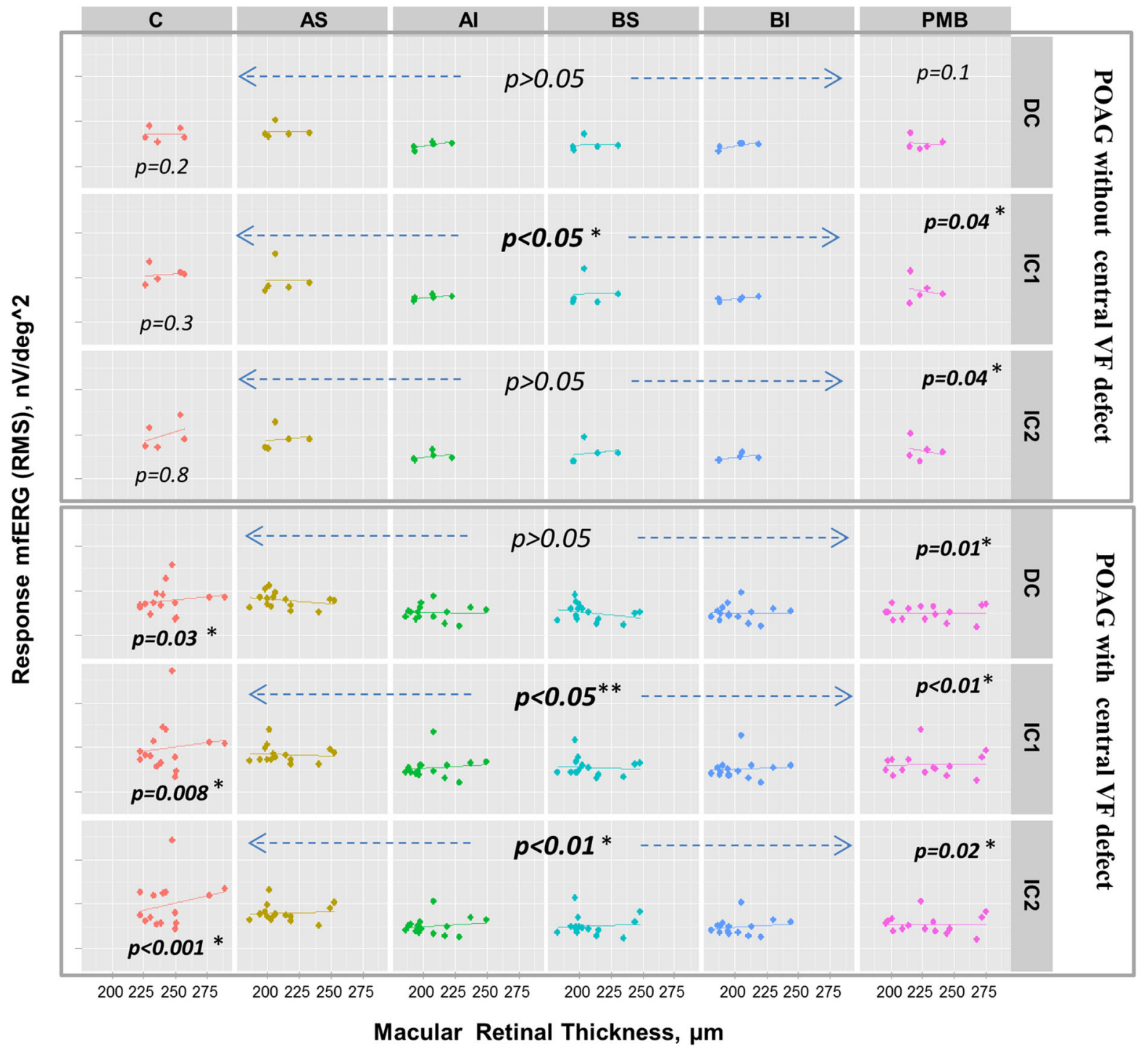

Fig. 3 Relation of mfERG RMS response to retinal macular thickness in two POAG subgroups (see text). $D C$ direct component, IC1 induced component 1, IC 2 induced component 2, $C$ central $10^{\circ}$ diameter, $A S$ and $A I$ superior and inferior

In the central, retinal ganglion cells are slightly displaced in their structural measurements when compared to functional tests $[9,15]$. The peak of the RGC lateral displacement occurs at the foveal border, at an eccentricity of about $2.5^{\circ}$ where it is about $0.6 \mathrm{~mm}$ [38]. The RGC lateral displacement drastically decreases out to $8.6^{\circ}$ eccentricity [39]. At the border of our $10^{\circ}$ central average area, the displacement of the RGC is only about $0.2 \mathrm{~mm}$; at the border of our $20^{\circ}$ area average, it is $<0.1 \mathrm{~mm}$ [39]. macular, $P M B$ papillomacular bundle area, $B S$ and $B I$ superior and inferior macula without PMB. *Significant after false discovery rate (FDR) adjustment. $* * p<0.057$ after false discovery rate (FDR) adjustment

In our study, we did not adjust for RGC displacement as values of functional tests (mfERG and perimetry) as well as of macular thickness were averaged over different areas as shown in Figs. 1 and 2. For the central area of largest displacement, values from the central $10^{\circ}$ were averaged. This area contains the maximal density of RGCs [39] and has only a very small displacement of $<0.2 \mathrm{~mm}$, which corresponds to $<0.7^{\circ}$. This seems negligible, especially, as we are 
measuring total macular thickness and not the ganglion cells/nerve fibre layer alone.

Correlation between macular thickness and visual fields

Most research in this area compares the morphological changes seen on OCT to functional assessments with automated perimetry [3, 9, 11, 29, 40-43]. For example, Leite et al. reported a moderate correlation between OCT and VF in advanced glaucoma. In early glaucoma or particularly in glaucoma suspects, this correlation appears weaker. This is in agreement with our findings where the correlation between OCT and VF macular sensitivity was stronger in the group of patients with advanced POAG. On the other hand, it has been reported that in advanced glaucoma, once a certain level of visual field damage has been obtained, measurement of RNFL thickness will be stable despite deterioration of visual fields due to remaining glia and blood vessels whose measurement is included in the OCT's measurement of the RNFL thickness [44].

Indeed, a residual RNFL thickness of about $45 \mu \mathrm{m}$ can be measured in eyes blind from glaucoma [45]. In their work, Leite et al. confirmed a model Hood et al. [29] had introduced a few years earlier. Hood et al. also described a weak correlation between the VF and the RNFL thickness for upper and lower arcuate defects. In addition, Hood et al. commented on the variability of the normal RNFL thickness ranging between 114.6 and $182.2 \mu \mathrm{m}$ in healthy subjects. This raises important issues for the diagnosis of glaucoma through assessment of the RNFL: in a person starting out with a thick RNFL, early glaucomatous dysfunction would be expected to appear as a significant SAP sensitivity loss prior to occurrence of a statistically significant OCT RNFL loss. The opposite would be the case in someone starting out with a thinner RNFL.

Correlation between macular thickness and electroretinography

Little has been reported about the correlation between electrophysiological methods such as pattern electroretinography (PERG) [46, 47] or multifocal visual evoked potential (mfVEP) [8] and morphological methods such as the OCT. Ventura et al. [48] reported that PERG amplitude and RNFL thickness significantly decrease with increasing severity of glaucoma.
However, in early glaucoma, this correlation is only weak. PERG is known to be sensitive to glaucomatous dysfunction and has been reported as abnormal in glaucoma even in the absence of central VF defects [49]. In addition, the PERG appeared more sensitive than the measure of peripapillary RNFL thickness [49]. In glaucoma patients with a central VF defect, there was a good correlation between the macular retinal thickness measured with the OCT and macular function assessed with VF and with the mfVEP [10]. Nevertheless, $8 \%$ of the eyes with abnormal VF and abnormal mfVEP had a normal OCT.

There are little data relating the mfERG to VF and OCT in glaucoma. Recently, Hori et al. [25] compared the mfERG obtained according to the ISCEV standard to macular OCT. They observed a weak but significant negative correlation between the nasal/temporal amplitude ratio of the secondorder kernel responses and the OCT in glaucoma. Patients with PPG were not studied. In experimental glaucoma in monkeys, Luo et al. [16] reported a significant correlation between local visual sensitivity and a low-frequency component of the mfERG that normally peaks at $12.1 \mathrm{~Hz}(\mathrm{SD} \pm 1.1$ and was dramatically reduced in experimental glaucoma. In another other study with experimental glaucoma (non-human primate model), high-frequency components of the mfERG were reduced prior to onset of optic nerve head surface topography change (measured by confocal scanning laser tomography) $(p<0.01)$ and prior to the onset of thinning of the peripapillary RFNL (measured on OCT) [50].

In our study, the induced components (ICs) of the mfERG correlated significantly with macular thickness. In patients with a central field defect, this was observed in all macular averages analysed. Early glaucoma patients, where 4 out of 5 had a PPG, showed a weaker, but still significant association between the ICs of the two-global flash mfERG and macular thickness outside the central $10^{\circ}$. In the central $10^{\circ}$, DC was associated significantly with macular thickness only in advanced glaucoma.

Onset of structural and functional changes in glaucoma/preperimetric glaucoma

In preperimetric glaucoma, clinically detectable optic nerve head changes are observed without detectable 
visual field damage. Retinal ganglion cell damage occurs in the central macula even in patients with normal VF (Humphrey 24-2) [40]. It has been reported that the detection of sensitivity loss by automated perimetry requires the loss of twice as many cells in the central area compared to the more peripheral retina [51].

The mfERG can also detect glaucomatous damage in PPG patients. In our preliminary study applying the two-global flash mfERG in PPG patients, we showed that the mfERG RMS of the central $10^{\circ}$ was significantly lower in PPG patients than in controls ( $p<0.01$ for all three epochs of the two-global flash mfERG (Ledolter AA et al. \#ARVO2013: A0126). However, as this is PPG, their VF is normal and so it is to be expected that the mfERG, if affected, will not correlate with any of analysed VF parameters.

When we divided our subjects into two subgroups depending on the presence of VF defects within the central $20^{\circ}$, mfERG-induced components correlated to linear macular cluster sensitivity in the central $10^{\circ}$ in patients with a central VF defect, but not in other locations. Macular thickness did not correlate to the VF parameters in our early glaucoma patients, whereas in moderate glaucoma, this correlation was highly significant.

\section{Structure-function correlation models}

Different structure-functional models were developed to evaluate the relationship between the structural and functional tests in the course of the glaucoma. Hood and Kardon defined the relationship between VF sensitivity in linear units and RNFL thickness in $\mu \mathrm{m}$ using the simple linear structure-function model and reported a residual RNFL thickness about $44-60 \mu \mathrm{m}$ in eyes with zero VF sensitivity. Using this model, the authors postulated that SAP loss, as well as OCT thickness, decreases linearly with RGC axon loss, where the non-neuronal thickness (glial cells and blood vessels) stays constant across age and stages of disease [29]. A curvilinear relationship has been reported between VF loss measured in $\mathrm{dB}$ and neuroretinal rim area. A logarithmic scale amplifies the sensitivity changes at the level of low decibels [28]. Thus, in early POAG neuroretinal rim area, changes appear greater than visual sensitivity loss, whereas in more advanced disease, it appears as if function changes at a greater rate than structure [28]. The unit of decibel is commonly used to express measured sensitivity in the nonlinear logarithmic scale. A 3-dB change in sensitivity means a doubling or halving of light intensity for detection. When VF sensitivity is plotted in linear units and spatial summation effects are accounted for, the structure-function relationship becomes more linear [28]. Electrophysiological studies of the relations between visual field sensitivity and PERG and mfVEP support the linear model of structure-function relationship in glaucoma [28, 30]. Considering PPG, it is possible to think about the socalled functional latency period that is the functional reserve in POAG. However, Garway-Heath et al. [28] reported a continuous (linear) relationship between functional tests (PERG amplitude and perimetry in linear units) and structural test (measure of neuroretinal rim area) with no ganglion cell functional reserve. Drasdo et al. [52] described the model of structurefunction relation where ganglion cell receptive field densities related linearly to the visual field sensitivity for the values $<29 \mathrm{~dB}$ and nonlinearly at higher sensitivities. The nonlinear model was developed by Harwerth et al. [53] on experimental glaucoma in monkey eyes based on a linear relationship between VF sensitivity and ganglion cell density if both parameters are plotted in decibel units. In the nonlinear model relationship between SAP sensitivity and RGC, density varies with retinal eccentricity and depends on the age and the stage of disease. Clinical application of this model produced correlated relationships between VF sensitivity and OCT thickness in normal eyes and eyes with early glaucoma and did not suggest that either significant structural defects precede functional or opposite functional defects precede structural [53].

\section{Papillomacular bundle area in glaucoma}

The special feature of the papillomacular bundle area has been noted by other researchers. Chen et al. [54] reported a reduction in papillomacular bundle thickness on the time-domain OCT in glaucoma patients even when the visual acuity remained good. Later Kita et al. [55] found that the RNFL thickness calculated in a sector corresponding to the papillomacular bundle was spared in early glaucoma. The authors did not find any significant decrease in RNFL when the thinning of macular ganglion cell complex was observed. By analysing this area, we, however, observed a significant negative association between the OCT and IC1 and IC2 
of mfERG in early glaucoma. In advanced glaucoma, this association was stronger. Macular thickness measured with td-OCT correlated significantly to the VF parameters in PMB only in moderate glaucoma.

\section{Limitation of this study and suggestions for further research}

Several problems arise when attempting a structurefunction analysis.

In choosing points of comparison, one needs to be aware that especially in the fovea, retinal ganglion cells are slightly displaced in their structural measurements when compared to functional tests $[9,15]$.

While for the areas analysed in this study, this seems of minor influence, it remains to be seen, if a more precise adjustment of the RGC displacement as well as an increase the number of the VF test points might improve the significance in structure-function relation in glaucoma [9].

Also the units of measure used, for instance, linear versus logarithmic, may influence the apparent association between structure and function [56]. In our analysis, VF sensitivity measured in linear range showed a significant association with induced components of mfERG in central $10^{\circ}$. This is in consensus with other reports $[28,32]$ that perimetric sensitivity in linear units is linearly related to ganglion cell density in tested regions of the retina. The induced components of two-global flash mfERG also relate to the function of inner retina, i.e. RGC.

Also, a limitation of the current study is that with the time-domain OCT only the entire retinal layer can be measured unless a segmentation technique is applied. However, in glaucoma, only the RGC and the RNFL are affected [3]. Thus, measurement of local RGC layer thickness alone could lead to a higher sensitivity of macular OCT measures in glaucoma and thus to an improved structure-function relationship in glaucoma. This sensitivity might be further increased with the help of fd-OCT, which has a higher resolution. The advantages of such a segmentation based on fd-OCT images have been showed recently [26, 27]. For the fd-OCT, Talamini et al. [26] could show that in patients with abnormal VF and abnormal mfERGs but normal appearing OCTs, manual segmentation showed 15 of 25 previously "normal" OCTs to be pathologic.
In summary, in this exploratory study, we studied the association between mfERG, td-OCT and SAP. The mfERG showed a positive association with total macular thickness in the central $10^{\circ}$. This association was stronger for IC1 and IC2 components of the twoglobal flash mfERG, mfERG components, which represent inner retinal function. In advanced glaucoma, ICs showed a significant association also in upper and lower $10^{\circ}-20^{\circ}$ hemifields. At the same time, macular thickness showed a strong positive association with visual field sensitivity in advanced glaucoma patients. When analysed in the linear range, macular sensitivity was significantly associated with the induced components of the mfERG in the central $10^{\circ}$. The two-flash multifocal ERG could thus be an additional tool to detect functional changes, especially in early stages of glaucoma, even prior to the occurrence of field defects.

Acknowledgments This work was supported by Swiss National Science Foundation (SNSF) 32003B-135219 (A.M.P.); LHW Stiftung Lichtenstein (A.M.P.).

Conflict of interest The author(s) have no proprietary or commercial interest in any materials discussed in the article.

\section{References}

1. Gupta N, Yücel YH (2007) Glaucoma as a neurodegenerative disease. Curr Opin Ophthalmol 18(2):110-114

2. Werkmeister RM, Cherecheanu AP, Garhofer G, Schmidl D, Schmetterer L (2013) Imaging of retinal ganglion cells in glaucoma: pitfalls and challenges. Cell Tissue Res 353(2): 261-268

3. Wang MHD, Hood DC, Cho JS, Ghadiali Q, De Moraes CG, Zhang X, Ritch R, Liebmann JM (2009) Measurement of local retinal ganglion cell layer thickness in patients with glaucoma using frequency-domain optical coherence tomography. Arch Ophthalmol 127(7):875-881

4. Atkin A, Bodis-Wollner I, Wolkstein M, Moss A, Podos SM (1979) Abnormalities of central contrast sensitivity in glaucoma. Am J Ophthalmol 88(2):205-211

5. Adams AJ, Heron G, Husted R (1987) Clinical measures of central vision function in glaucoma and ocular hypertension. Arch Ophthalmol 105(6):782-787

6. Lahav K, Levkovitch-Verbin H, Belkin M, Glovinsky Y, Polat U (2011) Reduced mesopic and photopic foveal contrast sensitivity in glaucoma. Arch Ophthalmol 129(1): $16-22$

7. Fujimoto JG, Drexler W, Schuman JS, Hitzenberger CK (2009) Optical coherence tomography (OCT) in ophthalmology: introduction. Opt Express 17(5):3978-3979

8. Parikh RS, Parikh SR, Thomas R (2010) Diagnostic capability of macular parameters of Stratus OCT 3 in detection of early glaucoma. Br J Ophthalmol 94(2):197-201 
9. Hood DC, Raza AS, de Moraes CG, Odel JG, Greenstein VC, Liebmann JM, Ritch R (2011) Initial arcuate defects within the central $10^{\circ}$ in glaucoma. Invest Ophthalmol Vis Sci 52(2):940-946

10. Kanadani FN, Hood D, Grippo T, Wangsupadilok B, Harizman N, Greenstein VC, Liebmann JM, Ritch R (2006) Structural and functional assessment of the macular region in patients with glaucoma. Br J Ophthalmol 90:1393-1397

11. Hood DC, Raza AS, de Moraes CGV, Johnson CA, Liebmann JM, Ritch R (2012) The nature of macular damage in glaucoma as revealed by averaging optical coherence tomography data. Trans Vis Sci Tech 1(1):3:1-3:15

12. Kim NR, Hong S, Kim JH, Rho SS, Seong GJ, Kim CY (2013) Comparison of macular ganglion cell complex thickness by Fourier-domain OCT in normal tension glaucoma and primary open-angle glaucoma. J Glaucoma 22(2):133-139

13. Hare W, Ton H, Woldemussie E, Ruiz G, Feldmann B, Wijono M (1999) Electrophysiological and histological measures of retinal injury in chronic ocular hypertensive monkeys. Eur J Ophthalmol 9(Suppl 1):30-33

14. Raz D, Seeliger MW, Geva AB, Percicot CL, Lambrou GN, Ofri R (2002) The effect of contrast and luminance on mfERG responses in a monkey model of glaucoma. Invest Ophthalmol Vis Sci 43(6):2027-2035

15. Frishman LJ, Saszik S, Harwerth RS, Viswanathan S, Li Y, Smith EL III, Robson JG, Barnes G (2000) Effects of experimental glaucoma in macaques on the multifocal ERG. Multifocal ERG in laser-induced glaucoma. Doc Ophthalmol 100(2-3):231-251

16. Luo X, Patel NB, Harwerth RS, Frishman LJ (2011) Loss of the low-frequency component of the global-flash multifocal electroretinogram in primate eyes with experimental glaucoma. Invest Ophthalmol Vis Sci 52(6):3792-3804

17. Luo X, Patel NB, Rajagopalan LP, Harwerth RS, Frishman LJ (2014) Relation between macular retinal ganglion cell/ inner plexiform layer thickness and multifocal electroretinogram measures in experimental glaucoma. Invest Ophthalmol Vis Sci 55(7):4512-4524

18. Todorova MG, Palmowski-Wolfe AM (2011) MfERG responses to long-duration white stimuli in glaucoma patients. Doc Ophthalmol 122(2):87-97

19. Sutter EE, Bearse MA, Shimada Y, Li Y (1999) A multifocal ERG protocol for testing retinal ganglion cell function. Invest Ophthalmol Vis Sci S15

20. Palmowski AM, Allgayer R, Heinemann-Vernaleken B, Ruprecht KW (2002) Multifocal ERG (MFERG) with a special multiflash stimulation technique in open angle glaucoma. Ophthalmic Res 34:83-89

21. Palmowski-Wolfe AM, Todorova MG, Orguel S, Flammer J, Brigell M (2007) The 'two global flash' mfERG in high and normal tension primary open-angle glaucoma. Doc Ophthalmol 114:9-19

22. Fortune B, Bearse MAJ, Cioffi GA, Johnson CA (2002) Selective loss of an oscillatory component from temporal retinal multifocal ERG responses in glaucoma. Invest Ophthalmol Vis Sci 43:2638-2647

23. Chu PHW, Chan HHL, Yiu-Fai Ng, Brown B, Siu AW, Beale BA, Gilger BC, Wongd F (2008) Porcine global flash multifocal electroretinogram: possible mechanisms for the glaucomatous changes in contrast response function. Vis Res 48:1726-1734

24. Kramer SA, Ledolter AA, Todorova MG, Schötzau A, Orgül S, Palmowski-Wolfe AM (2013) The 2-global flash mfERG in glaucoma: attempting to increase sensitivity by reducing the focal flash luminance and changing filter settings. Doc Ophthalmol 126(1):57-67

25. Hori N, Komori S, Yamada H, Sawada A, Nomura Y, Mochizuki K, Yamamoto T (2012) Assessment of macular function of glaucomatous eyes by multifocal electroretinograms. Doc Ophthalmol 4(125):235-247

26. Talamini CL, Raza AS, Dale EA, Greenstein VC, Odel JG, Hood DC (2011) Abnormal multifocal ERG findings in patients with normal-appearing retinal anatomy. Doc Ophthalmol 123(3):187-192

27. Villoslada P, Cuneo A, Gelfand J, Hauser SL, Green A (2012) Color vision is strongly associated with retinal thinning in multiple sclerosis. Mult Scler 18(7):991-999

28. Garway-Heath DF, Holder GE, Fitzke FW, Hitchings RA (2002) Relationship between electrophysiological, psychophysical, and anatomical measurements in glaucoma. Invest Ophthalmol Vis Sci 43:2213-2220

29. Hood D, Kardon R (2007) A framework for comparing structural and functional measures of glaucomatous damage. Prog Retin Eye Res 26(6):688-710

30. De Moraes CG, Liebmann JM, Ritch R, Hood DC (2012) Clinical use of multifocal visual-evoked potentials in a glaucoma practice: a prospective study. Doc Ophthalmol 125(1):1-9

31. Dale EA, Hood DC, Greenstein VC, Odel JG (2010) A comparison of multifocal ERG and frequency domain OCT changes in patients with abnormalities of the retina. Doc Ophthalmol 120:175-186

32. Malik R, Swanson WH, Garway-Heath DF (2012) Structure-function relationship' in glaucoma: past thinking and current concepts. Clin Exp Ophthalmol 40(4):369-380

33. Hood D, Harizman N, Kanadani FN, Grippo TM, Baharestani S, Greenstein VC, Liebmann JM, Ritch R (2007) Retinal nerve fibre thickness measured with optical coherence tomography accurately detects confirmed glaucomatous damage. Br J Ophthalmol 91:905-907

34. Medeiros FA, Zangwill LM, Bowd C, Vessani RM, Susanna R Jr, Weinreb RN (2005) Evaluation of retinal nerve fiber layer, optic nerve head, and macular thickness measurements for glaucoma detection using optical coherence tomography. Am J Ophthalmol 139:44-55

35. Weijland A, Fankhauser F, Bebie H, Flammer J (2004) Automated perimetry. Visual field digest, 5th edn. HaagStreit AG, Switzerland, p 61

36. Palmowski-Wolfe A (2012) Can the OCT replace functional tests such as the mfERG? Invest Ophthalmol Vis Sci 53(10):6129

37. Lee K, Lee J, Na J, Kook M (2013) Usefulness of macular thickness derived from spectral-domain optical coherence tomography (SD-OCT) in the detection of glaucoma progression. Invest Ophthalmol Vis Sci 54(3):1941-1949

38. Drasdo N, Millican CL, Katholi CR, Curcio CA (2007) The length of Henle fibers in the human retina and a model of ganglion receptive field density in the visual field. Vis Res 47(22):2901-2911 
39. Sjöstrand J, Popovic Z, Conradi N, Marshall J (1999) Morphometric study of the displacement of retinal ganglion cells subserving cones within the human fovea. Graefes Arch Clin Exp Ophthalmol 237(12):1014-1023

40. Raza AS, Cho J, de Moraes CG, Wang M, Zhang X, Kardon RH, Liebmann JM, Ritch R, Hood DC (2011) Retinal ganglion cell layer thickness and local visual field sensitivity in glaucoma. Arch Ophthalmol 129(12):1529-1536

41. Pinto LM, Costa EF, Gross PB, Kavay MM, Fogaça L, Melo LAS Jr, Paranhos A Jr (2010) Evaluation of macular structure and function in glaucoma. Invest Ophthalmol Vis Sci 51:E-Abstract 4913

42. Zhang X, Bregman CJ, Raza AS, De Moraes G, Hood DC (2011) Deriving visual field loss based upon OCT of inner retinal thicknesses of the macula. Biomed Opt Express 2(6):1734-1742

43. Hood DC, Raza AS (2011) Method for comparing visual field defects to local RNFL and RGC damage seen on frequency domain OCT in patients with glaucoma. Biomed Opt Express 5(2):1097-1105

44. Leite M, Zangwill L, Weinreb R, Rao H, Alencar L, Medeiros F (2012) Structure-function relationships using the Cirrus spectral domain optical coherence tomograph and standard automated perimetry. J Glaucoma 21(1):49-54

45. Sihota R, Sony P, Gupta V, Dada T, Singh R (2006) Diagnostic capability of optical coherence tomography in evaluating the degree of glaucomatous retinal nerve fiber damage. Invest Ophthalmol Vis Sci 47(5):2006-2010

46. Falsini B, Marangoni D, Salgarello T, Stifano G, Montrone L, Campagna F, Aliberti S, Balestrazzi E, Colotto A (2008) Structure-function relationship in ocular hypertension and glaucoma: interindividual and interocular analysis by OCT and pattern ERG. Graefes Arch Clin Exp Ophthalmol 246(8):1153-1162

47. Bowd C, Tafreshi A, Zangwill LM, Medeiros FA, Sample PA, Weinreb RN (2011) Pattern electroretinogram association with spectral domain-OCT structural measurements in glaucoma. Eye (Lond) 25(2):224-232

48. Ventura L, Sorokac N, De Los Santos R, Feuer W, Porciatti V (2006) The relationship between retinal ganglion cell function and retinal nerve fiber thickness in early glaucoma. Invest Ophthalmol Vis Sci 47(9):3904-3911

49. Bach M, Birkner-Binder D, Pfeiffer N (1990) Das Musterelektroretinogramm bei fruhem Glaukom und bei okularer Hypertension. Fortschr-Ophthalmol 87(6):591-593

50. Fortune B, Burgoyne CF, Cull GA, Reynaud J, Wang L (2012) Structural and functional abnormalities of retinal ganglion cells measured in vivo at the onset of optic nerve head surface change in experimental glaucoma. Invest Ophthalmol Vis Sci. 53(7):3939-3950

51. Quigley HA, Dunkelberger GR, Green WR (1989) Retinal ganglion cell atrophy correlated with automated perimetry in human eyes with glaucoma. Am J Ophthalmol 107: 453-464

52. Drasdo N, Mortlock KE, North RV (2008) Ganglion cell loss and dysfunction: relationship to perimetric sensitivity. Optom Vis Sci 85(11):1036-1042

53. Harwerth RS, Wheat JL, Fredette MJ, Anderson DR (2010) Linking structure and function in glaucoma. Prog Retin Eye Res 29(4):249-271

54. Chen E, Gedda U, Landau I (2001) Thinning of the papillomacular bundle in the glaucomatous eye and its influence on the reference plane of the Heidelberg retinal tomography. J Glaucoma 10(5):386-389

55. Kita Y, Kita R, Nitta A, Nishimura C, Tomita G (2011) Glaucomatous eye macular ganglion cell complex thickness and its relation to temporal circumpapillary retinal nerve fiber layer thickness. Jpn J Ophthalmol 55(3):228-234

56. Marín-Franch I, Malik R, Crabb DP, Swanson WH (2013) Choice of statistical method influences apparent association between structure and function in glaucoma. Invest Ophthalmol Vis Sci 54(6):4189-4196 